\title{
Derivation of Night Time Behaviour Metrics using Ambient Sensors
}

\author{
Andrea Kealy, Kevin McDaid ${ }^{1}$, John Loane, Lorcan Walsh, Julie Doyle \\ CASALA, ${ }^{1}$ STORC \\ Dundalk Institute of Technology, Dublin Road \\ Dundalk, Co. Louth, Ireland \\ \{andrea.kealy, john.loane, lorcan.walsh, julie.doyle\}@ casala.ie, kevin.mcdaid@dkit.ie
}

\begin{abstract}
Sleep problems have been shown to have significant negative impact on health. As such it is important to examine night time behaviour to objectively determine when sleep disturbances arise. Due to the large night-to-night variability in sleep quality for older adults, it is important to objectively measure behaviour over a significant period to establish trends or changes in patterns of sleep. In this paper we present a means of ambiently monitoring sleep through the use of sensors installed in each of sixteen independent living apartments. We investigate the effect of time outside the home and movement within the home on sleep. These measures are validated against comparative measures from two actigraph datasets. The first consisting of five adults, two of whom are healthy subjects and the other three adults have previously fallen, gathered over a period of between two and four nights. The second consisting of three older adults recorded over seven nights in their own homes. Results relating time outside the home and movement within the home to sleep are presented for three individuals spanning a period of between 630 and 650 days.
\end{abstract}

Keywords-AAL; aging in place; ambient monitoring; sleep measures

\section{INTRODUCTION}

Continuous monitoring can support the detection of early signs of age related illnesses, ensuring early intervention is provided [1, 2], to maintain wellbeing. In [3] it was established that subtle changes in the behaviour of a person can provide early indications of age related decline. The integration of unobtrusive, ambient sensors within an individual's living environment has been proposed as a means for facilitating such continuous monitoring. The large scale ambient setups required for such monitoring produce a vast dataset of unrefined data. Resultantly data analysis techniques are required to ensure such ambient monitoring is efficient and cost effective in providing the necessary monitoring required for older people to "age in place".

Degradation in sleep over time has been shown to have a considerable negative impact on health [4, 5]. Long term variation is difficult to measure and home-based studies typically are of short duration, particularly if contact-based sensors are used. With significant night-to-night variability being reported in older adults it is important that longitudinal changes are considered in determining if a significant decline in sleep quality has occurred. In this paper we describe the derivation of metrics by means of a location mapping model to

CASALA has been established and initially funded under the Enterprise Ireland Applied Research Enhancement (ARE) programme determine characteristics of sleep pattern including bed time; rise time; sleep disturbances and time in bed, from ambient sensors installed in sixteen independent living apartments. This model has been validated by comparison of the derived metrics with similar metrics established through actigraphy, the ambulatory gold standard for sleep monitoring. A longitudinal investigation of the variation in daily activity and sleep measures is then presented for three individuals residing in their homes, and compared to a range of subjective sleep quality scores, as determined by Pittsburgh Sleep Quality Index (PSQI) [6] questionnaire results.

The main contribution of this paper is the development, validation and implementation of an ambient night time behavior monitoring system that is particularly suited for extended deployments. Highly-validated algorithms extract specific nocturnal activity metrics including time in bed, time outside the home and total movement detected. Subsequently, these algorithms were applied to data collected from the permanent homes of older adults over an extended duration of between 630 and 650 days. The paper illustrates the use of ambient unobtrusive sensors to monitor longitudinal patterns of night time and sleep behavior and presents methods for the detection of medium term changes in behaviour over time.

The remainder of the paper is structured as follows. In section II an overview of the related research is given. In section III the methodology is provided. Results of a comparison between ambient metrics of night time behaviour and actigraphy-derived sleep metrics are presented in section IV. Results from a longitudinal analysis of time in bed are compared against detected movement and time outside the home metrics in section V. Limitations are discussed and conclusions are presented in section VI.

\section{RELATED RESEARCH}

The established real-world technology for sleep monitoring is wrist actigraphy, however this is unsuitable for certain populations and over extended durations. The development of an appropriate technology for long term sleep monitoring has been the focus of many research groups. Various non-contact technologies have been investigated including PIR-based systems [7, 8]; biomotion sensors using Doppler radar to detect sleep [9]; pressure-based systems such as the Under Mattress Bed Sensor (UMBS) [10], load cells discriminating Sleep Behaviour Disorder (SBD) from normal breathing [11, 
12], and pneumatic tubes to estimate physiological signals and infer various sleep metrics [13-15]. However such a system must be integrated into an intelligent environment to facilitate the long term collection of sleep related data.

Ongoing research in the Orcatech Living Lab [16] is investigating the monitoring of motion [17] and sleep patterns [18] in order to determine location, walking speed and daily activities. Unobtrusive monitoring technologies have been installed within the homes of community dwelling older adults, to provide a testbed for evaluating behaviour monitoring technology. This could support the detection of early changes in cognitive function and inform clinicians of a change in health status. CASAS smart home testbed [19] has been used to generate models of user behaviour as well as recognise activities. In [20] the authors present online activity recognition and discovery within three smart apartment testbeds over a six month period. The resulting information may be used in performing functional health assessments longitudinally and in prompting users in their activities of daily living as required. Work ongoing at University of Missouri (MU) [21-23], consists of analysing data from a 31 unit facility housing older people who would have otherwise made the move to a nursing home. Accordingly on-site support is provided from healthcare professionals, such as nurses and physical therapists. Maintenance, housekeeping, transportation and dining services are also provided and social activities are scheduled regularly. Their setup facilitates the detection of specific activities using PIRs and the measurement of restlessness in bed using bed sensors. It is intended to monitor and assess potential problems in mobility and cognition of older people in their homes and may be used in detecting falls, and changes in daily patterns which may indicate onset of a health problem [24].

\section{METHODOLOGY}

\section{A. Great Northern Haven Overview}

The Great Northern Haven (GNH) is a purpose-built demonstration housing project in Dundalk, Ireland consisting of sixteen independent living apartments. Each apartment has been fitted with a series of ambient sensors and actuators intended to monitor and support residents. The integrated sensors include passive infra-red (PIR) sensors to detect motion; contact sensors on all windows and exterior doors as well as on three interior doors; sensors on all light switches; temperature sensors within each room; brightness sensors in three rooms; and sensors to detect electricity and heating usage. Data is collected via a KNX infrastructure and stored in a MySQL database.

Fifteen apartments are permanently occupied, with residents aged 58 to 87 . Among the permanent residents of GNH one apartment is multiple occupancy, in which a married couple reside, with the remaining apartments being occupied singly, accommodating 10 men and 4 women. Residents suffer from a wide range of illnesses including heart conditions; diabetes; depression; bipolar disorder; with one resident having previously suffered a stroke and another having suffered from cancer. The sixteenth apartment is used as a demonstration/test apartment as well as being designated as a transition unit for falls prevention. Clinically validated questionnaires, administered every six months, subjectively monitor changes in physical and emotional wellbeing. Results from two questionnaires, Pittsburgh Sleep Quality Index (PSQI) [6] and the De Jong scale of emotional and social loneliness [25], are discussed in section V.

Our setup differs from those presented in section II in that residents live independently in a real world environment, integrated with monitoring technology which has been extensively validated.

\section{B. Sensor Validation}

Considerable validation has been carried out on all sensor data to ensure the integrity of the data is maintained. Days in which data loss occurred, as determined by strict quality measures [26], have been omitted from analysis. The location mapping model, detailed in section $\mathrm{C}$, relies on PIRs in determining the location of a resident. Consequently PIRs were evaluated to determine their visibility within each apartment. As apartment layout and sensor placement is consistent in all apartments in $\mathrm{GNH}$, validation within the test/demonstration apartment was valid for all apartments.

PIR validation was carried out in each room containing a PIR, as well as entrances to adjacent rooms in the line of sight of a PIR. These areas included the open plan living room and kitchen; the hallway, as well as entrances to the bathroom and guest bedroom; and finally the main bedroom and en suite. Rows of points were marked with tape every $35 \mathrm{~cm}$ parallel to the wall holding the PIR, see fig. 1. Continuous motion, from the waist up, was carried out on each point for the overshoot time (ten seconds) plus an additional five seconds to ensure that movement was measured on each point. This method was performed on all points parallel to the PIR bearing wall, following the red lines in fig. 1, and also diagonally, along the blue lines. The blue areas of figs. 1 and 2 show areas that remain undetected by PIRs. Areas which detect movement towards them but don't detect subtle movement within them, i.e. the PIR fired when moving to the spot but then shut off after a few seconds, are coloured light green. Areas completely visible to the PIR sensor, are shown in dark green.

Detection of movement into and out of the bedroom is a requirement in determining the sleep window, described in section D. Results have indicated that PIRs should be positioned at a transition point between rooms in order to

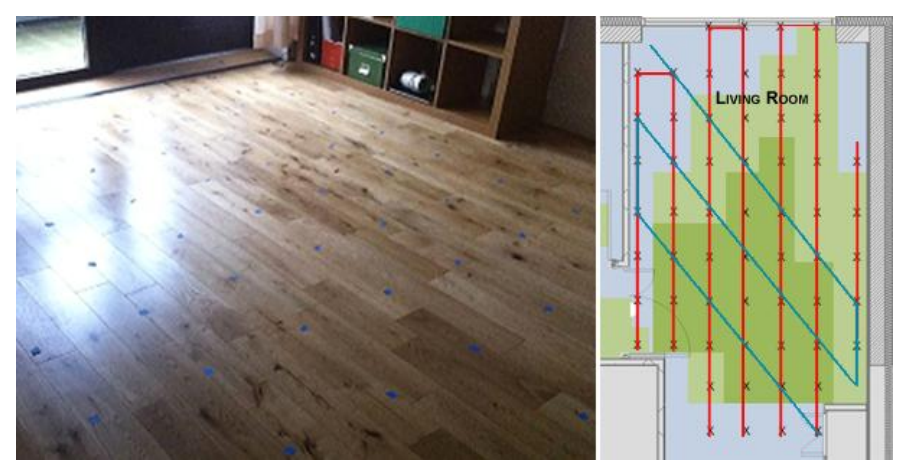

Figure 1. GNH floor validation markers (left) and path of floor validation (right) within the living room 

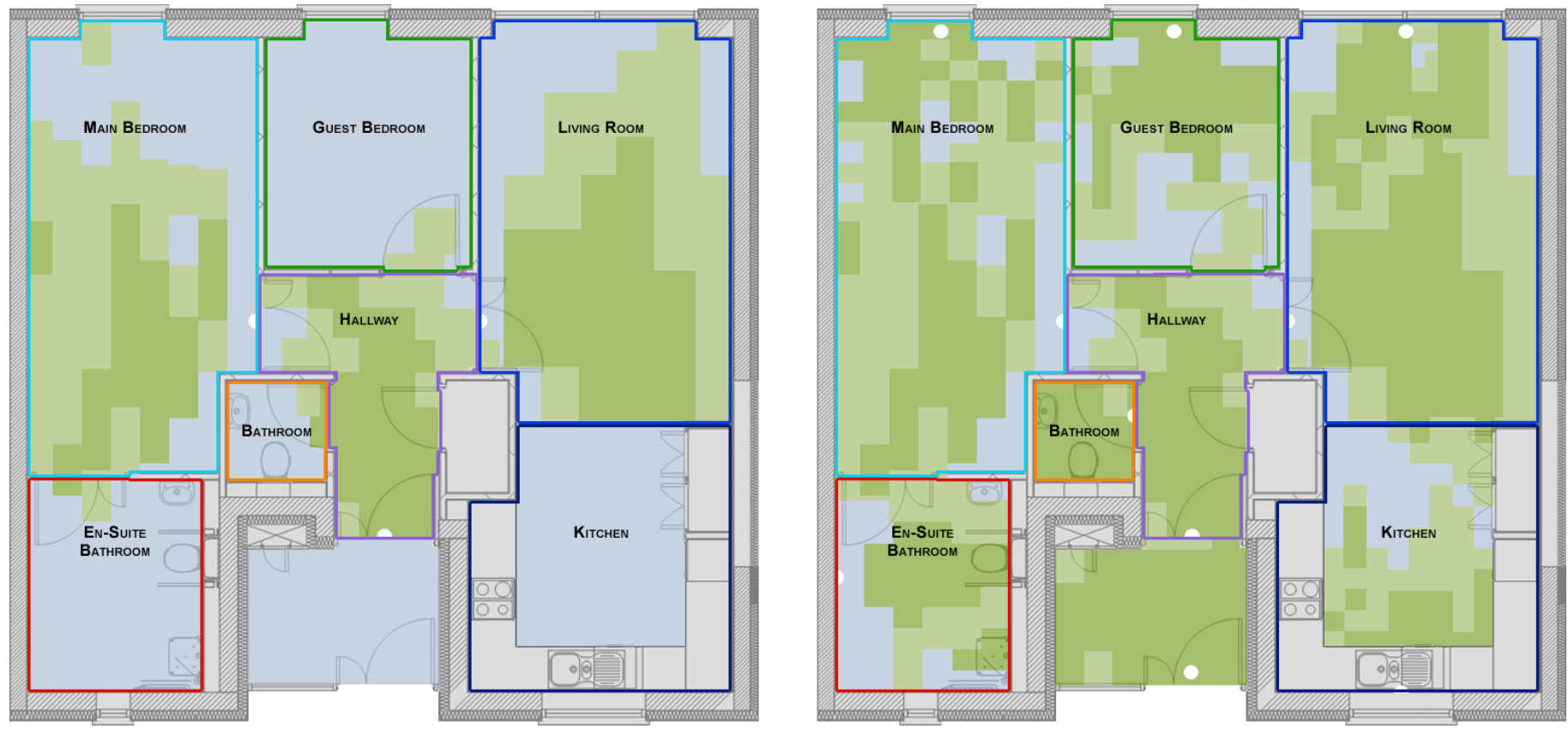

Figure 2. Standard PIR visibility in GNH apartments (a); visibility with additional PIR sensors (b), as described in section III.B (overlaid location mapping zones, as described in section III.C)

accurately determine location. Fig. 2(a) illustrates the standard placement and visibility of PIRs located in the hallway, living room and main bedroom of GNH. A number of additional PIRs have been installed in the test/demonstration apartment at GNH to achieve a higher level of visibility. This increased visibility, illustrated in fig. 2(b), facilitates validation of the location mapping model discussed in section $\mathrm{C}$.

\section{Location Mapping}

A location mapping algorithm has been developed, using a subset of sensors installed in GNH. All PIRs have been validated to detect room transitions. Consequently it is possible to deduce when a resident is in a room in which a PIR

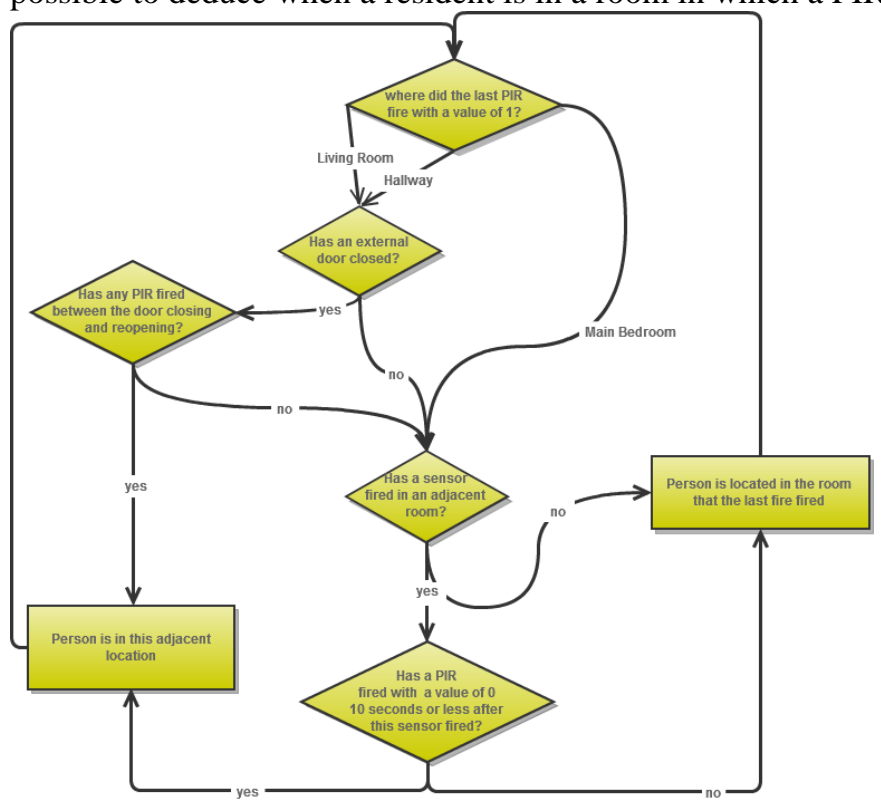

Figure 3. Location mapping model is installed. It is important to note that if PIRs were installed in each room, as is the case in the test/demonstration apartment depicted in fig. 2(b), additional sensors would not be required to establish the location of the resident. As this is not the case in the permanently occupied apartments within GNH the location mapping decision model, as illustrated in fig. 3, is used. This decision model is used to establish whether: 1) the resident remained in the room within which a PIR has most recently fired; or 2) the resident moved to an adjacent room in which movement is undetectable by the PIR. In GNH any room which does not have a PIR installed is adjacent to a room containing a PIR.

Movement to adjacent rooms, identified by the pale blue areas in fig. 2(a), is determined by checking for changed values of any "periodic" or "on change" sensor within adjacent rooms. A "periodic" sensor, such as a contact sensor on a window or door, is determined to have fired when a change in value is registered. An "on change" sensor is determined to have fired any time a value is registered. If a sensor is triggered in a room adjacent to the room in which the most recently fired PIR is located, a person is determined to be in the adjacent room between the time that PIR detected a cease in movement and the time it next detects movement. Adjacent rooms in GNH include the en suite, adjacent to the main bedroom; the bathroom, guest bedroom and outside, adjacent to the hallway; or the kitchen, adjacent to the living room.

This algorithm gives comparable results when evaluating the typical setup using three PIRs, shown in fig 2(a), against the setup using the additional PIRs, shown in fig 2(b), which offers almost full PIR visibility.

Two visits have been carried out to determine the validity of the location mapping algorithm. These visits took place in the test/demonstration apartment in GNH. Emerald Timestamp app for iPhone was used to record transitions between rooms 
as well as door and light switch usage. Derived location maps were analysed against the locations recorded using Emerald Timestamp during the visit. The first visit involved typical movement around the apartment. The second visit focused on testing for false firings. This involved moving into and out of adjacent rooms in which light switch sensors are located within line of sight of a PIR. These included the en suite, guest bedroom and bathroom.

In the first visit the location mapping algorithm was determined to consistently capture all movement to rooms in which PIRs are located, all time outside the home events and was successful in capturing all movement to adjacent rooms where doors and light switches were used. One false firing was detected in the kitchen after a switch to open/close the kitchen window was used followed directly by a period of no motion in the living room. This results from the switch being located in direct view of the PIR in the living room. Results of the second visit confirmed this issue. When the en suite light switch was switched on and movement into the en suite did not directly follow, movement to these adjacent rooms was either mislabelled or missed completely. This issue is resolved when sensors located in areas undetectable by PIRs are used in conjunction with sensors located in direct view of a PIR, for example when light switches and doors are used together, or doors are used on their own.

These false firings result from the added complexity introduced by the overshoot time on each PIR. In GNH all PIRs have an overshoot time of ten seconds. When a PIR initially detects motion, it immediately sets to a value of 1 and the overshoot time is started. Updates are not sent during the overshoot time. Once the overshoot time is over, it will either restart if motion has been detected, or reset the PIR value to a 0 . Consequently motion may be detected by a PIR ten seconds after a sensor in an adjacent room has been triggered.

\section{Sleep Measures}

Various sleep measures have been derived by considering elongated periods within the bedroom, as deduced by the location mapping algorithm. Metrics investigated include estimated bed time and rise time, sleep disturbances and total time in bed. Bedroom and out of bedroom events, as calculated by the location mapping algorithm, are examined in determining the estimated sleep window, i.e. the period within which the bed time and rise time are located. Periods spent out of the bedroom from 3am onwards are compared to the in bedroom event that follows. For example if an out of bedroom event occurs and is determined to be short, in comparison to the in bedroom event that follows, it is categorised as a sleep disturbance, is grouped within the current sleep window and the period that follows is investigated further. If however the duration spent outside the bedroom is greater than the following in bedroom event, the end of the previous period in the bedroom is deemed to be the end of the current sleep window. This is repeated in reverse to detect the start of the estimated sleep window.

\section{1) Bed Time (BT) and Rise Time (RT)}

Once an estimated sleep window is determined for a given night the movement levels, as determined by the PIR in the bedroom, at the beginning of this sleep window are used to estimate the bed time and rise time. Movement is segmented into 1 minute epochs. The bed time is determined as the start of a period where movement is below a subject-specific movement threshold for at least 10 epochs. This threshold is based on normal nightly movement levels of an individual in their first month residing in GNH. As movement may reduce to a level below their threshold as a result of reading in the bedroom, a secondary requirement is in place to verify that the main bedroom light is switched off before estimating bed time. Rise time is estimated by deducing the last period at the end of the sleep window whereby movement is below a subjectspecific threshold for at least 10 epochs.

\section{2) Sleep Disturbances (SD)}

A sleep disturbance is determined by a change in location, or a grouping of epochs in which movement is above the movement threshold, between the estimated bed time and rise time. Where changes in location occur the rules for determining bed time and rise time are applied to calculate the estimated wake time before the disturbance and the estimated sleep time after the disturbance. The total disturbance time is calculated as the duration, in hours, between the estimated wake time and the sleep time.

\section{3) Time in Bed (TIB)}

Time in bed is calculated as the duration, in hours, between bed time and rise time, removing periods which were calculated to be sleep disturbances.

\section{E. Activity Measures}

The relationship between time outside the home and total movement on sleep is unknown, however changes in these activity measures may have an impact on sleep over time, this is investigated further in section V.

\section{1) Time Outside the Home (TOTH)}

A person is determined as being outside the home when no movement is detected between an exterior door closing and being re-opened. It may be calculated by subtracting the time a person closes an external door from the time they re-open the external door, providing no PIR has fired with a value of 1 during this period.

\section{2) Total Movement (TM)}

The total movement is calculated as the time between a PIR firing with a value of 1 and the time the PIR resets to a 0 value. As all PIRs in GNH have an overshoot time of ten seconds, this overshoot time is removed from the result. Total movement is segmented into 1 minute bins.

\section{COMPARISON OF NigHT-TIME BEHAVIOUR METRICS}

\section{A. Experiment Setup}

A wrist-worn tri-axial actigraph (MotionWatch 8, CamNtech, Cambridge, UK) was used to validate night time activity metrics. The actigraph was set to record movement and light levels, recorded as luminous intensity (lux) of white light, in 30 second epochs. Participants were asked to use the event marker button to indicate when they were going to bed and getting up. Metrics derived by our algorithms namely rise 
time; bed time and time in bed were evaluated against comparable metrics such as "fell asleep", "woke up" and "actual sleep" derived using the software that accompanied the actigraph. Each participant was requested to fill out a sleep diary daily and a Pittsburgh Sleep Quality Index (PSQI) questionnaire was administered once for each participant during the validation period to determine their sleep quality score.

Two groups participated in the validation. Group A consisted of five individuals staying in the test/demonstration apartment over a period of between two and four nights. Three of group A were older adults, two female and one male, who had previously fallen and two adults, one female and one male, who were healthy. Group B consisted of three male GNH residents, aged between 62 and 87, over a period of between seven and ten nights within their own homes.

Sensor derived measures for time in bed (TIB-S) were compared to equivalent measures derived from the actigraph data (TIB-A) and daily sleep diaries (TIB-SR). Movement was compared in 1 minute bins and the results normalised. Details are given in Table I.

\section{B. Results}

The correlation between TIB-S and TIB-A for group A, shown in table I, was found to be strong, ranging from 0.71 to 1. A strong correlation, $0.98,0.97$ and 1 respectively, was recorded when evaluating TIB-S data against TIB-A for group B. In contrast the TIB-SR showed a lower correlation of 0.9, 0.35 and -0.1 respectively when correlating against TIB-S, and $0.9,0.3$ and 0.14 respectively when correlating against TIB-A for group B. Figs. 4, 5 and 6 illustrate the movement levels of group B, generated from the three PIRs (black), as well as raw movement data, generated from the actigraph (gray), overlaid on location maps.

TABLE I. COMPARISON OF TIME IN BED DERIVED FROM SENSORS; ACTIGRAPHS; AND SELF-REPORTS

\begin{tabular}{|c|c|c|c|c|c|c|c|c|}
\hline 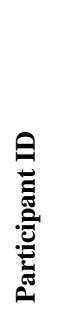 & $\underset{4}{4}$ & ڤ્ & 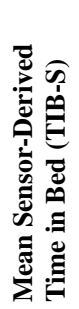 & 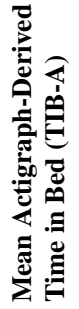 & 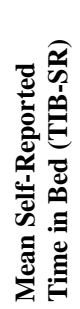 & 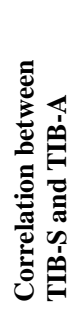 & 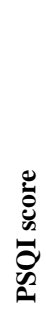 & 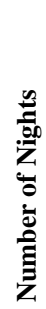 \\
\hline $1 \mathrm{~A}$ & 76 & F & 9.68 & 9.40 & 12.75 & 0.93 & 14 & 4 \\
\hline $2 \mathrm{~A}$ & 78 & M & 5.50 & 6.21 & 9.25 & 0.76 & 6 & 4 \\
\hline $3 \mathrm{~A}$ & 66 & F & 7.65 & 8.04 & 8.50 & 0.71 & 10 & 3 \\
\hline $4 \mathrm{~A}$ & 31 & $\mathrm{~F}$ & 7.68 & 7.57 & 8.00 & 0.97 & 1 & 4 \\
\hline $5 \mathrm{~A}$ & 28 & M & 9.06 & 9.06 & 8.50 & 1.00 & 1 & 2 \\
\hline $1 \mathrm{~B}$ & 62 & $\mathrm{M}$ & 7.79 & 6.71 & 8.76 & 0.98 & 6 & 7 \\
\hline $2 B$ & 87 & M & 9.41 & 8.26 & 5.83 & 0.97 & 12 & 6 \\
\hline $3 \mathrm{~B}$ & 70 & M & 10.35 & 10.20 & 9.25 & 0.97 & 1 & 2 \\
\hline
\end{tabular}

Time in Bed (TIB) is measured in hours

\section{Discussion and Conclusions}

Results for group A show close alignment between TIB-S and TIB-A in contrast to the evident difference when correlating TIB-SR against both TIB-S and TIB-A. The lower correlations of 0.76 and 0.71 , for this group, result from delayed sleep start times and increased sleep disturbances being reported. This resulted from a configuration change which increased the overshoot time for the PIR within the main bedroom from ten seconds to ten minutes. The error affected two days of data for participant $1 \mathrm{~A}$ and remained an issue for the full duration which participant $2 \mathrm{~A}$ and $3 \mathrm{~A}$ resided. Data for participant $4 \mathrm{~A}$ and $5 \mathrm{~A}$ were unaffected.

In examining location maps for group B, very few signs of actigraph movement, represented in gray, are evident for participant $2 \mathrm{~B}$ on night 1 , illustrated in fig. 5. The participant appeared to have taken off the watch, resulting in 6 nights being analysed for this participant. Likewise, participant 3B appeared to remove the actigraph in days 1-7, as evident in fig. 6. Additionally the location map appeared to have mislabelled en suite events on the morning of day 10, with two events appearing in close proximity. This resulted in only two nights being used in the analysis for participant 3B.

Time outside the home events and durations spent in areas undetected by PIRs may be recognised visually by identifying lengths of time where movement is detected by the actigraph (gray) but not by PIRs (black). This is particularly evident in fig. 4 during times spent outside the home (white) and times spent in the en suite (red). Some instances are evident where the location map is registering presence within a room containing a PIR despite movement being undetected by the PIRs. This may result from a resident being in an area of a room undetectable to PIRs, or being in an adjacent room without using sensors whilst in this adjacent room. This is evident for participant $1 \mathrm{~B}$ on day 1 around 10:30pm where

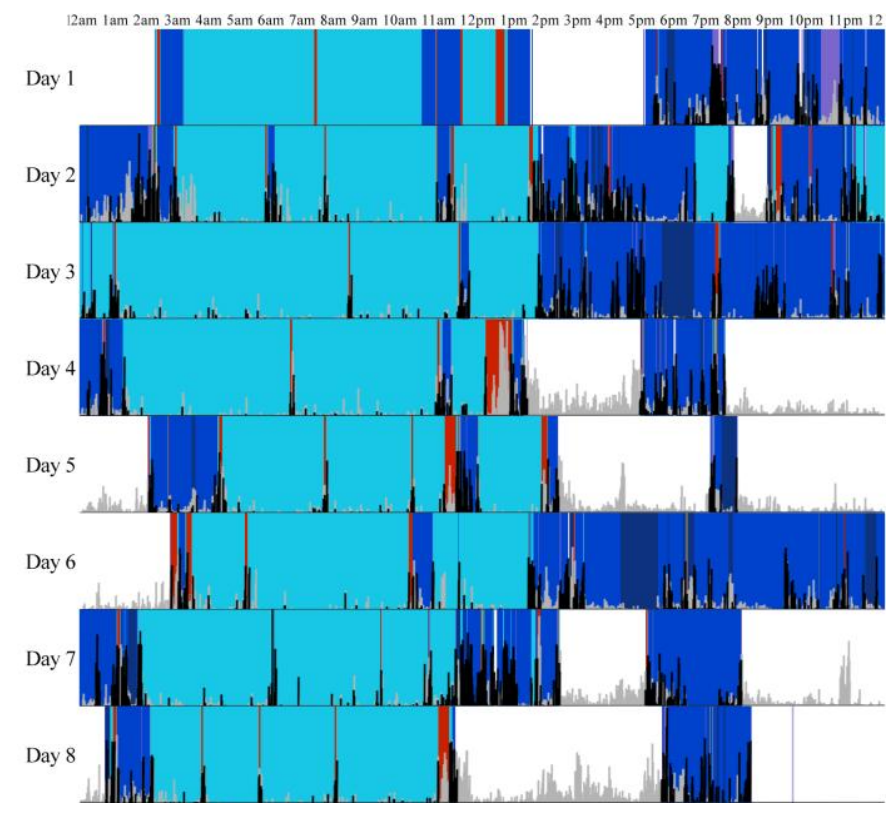

Figure 4. Location map for participant 1B, a poor sleeper (PSQI=6) [living room (blue); kitchen (navy); main bedroom (aqua); en suite (red); hallway (purple); bathroom (orange); and time outside the home (white). Sensor (black) and actigraph (gray) movement levels are overlaid] 


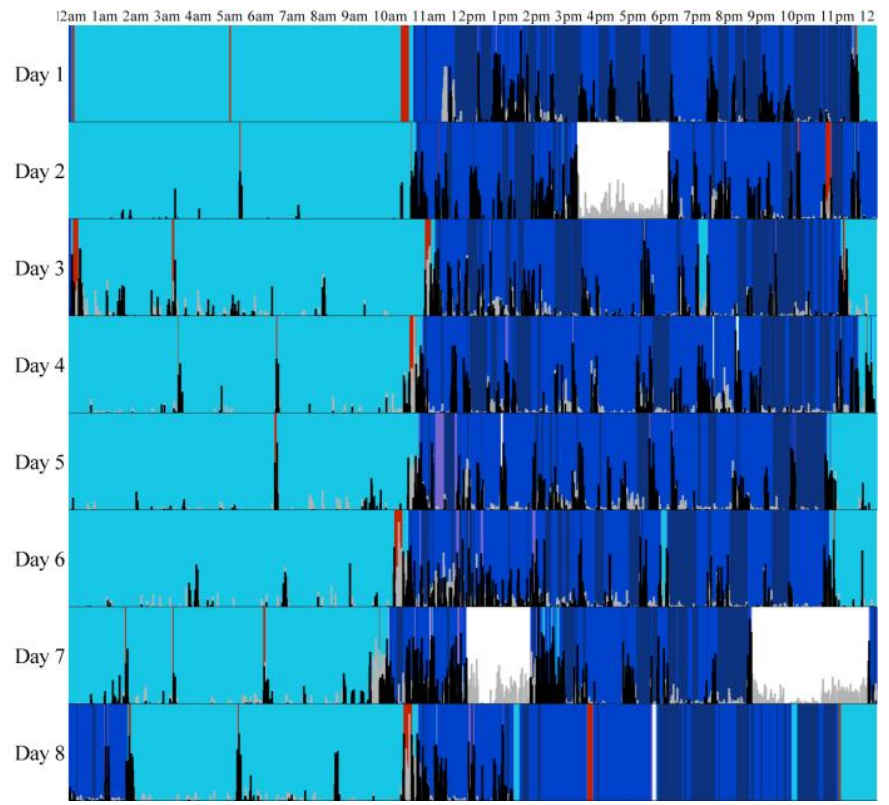

Figure 5. Location map for participant 2B, a poor sleeper (PSQI=12)

movement is detected by the actigraph while the resident is in the hallway. This could result from the resident being in the bathroom or guest bedroom, or alternatively being away from home (if external doors were not fully closed).

The location maps depict the diverse way in which each resident uses their home. Whilst both participant 2B and 3B spend a long time in the bedroom at night, participant $2 \mathrm{~B}$ has a very consistent routine, participant $3 \mathrm{~B}$ has considerably less

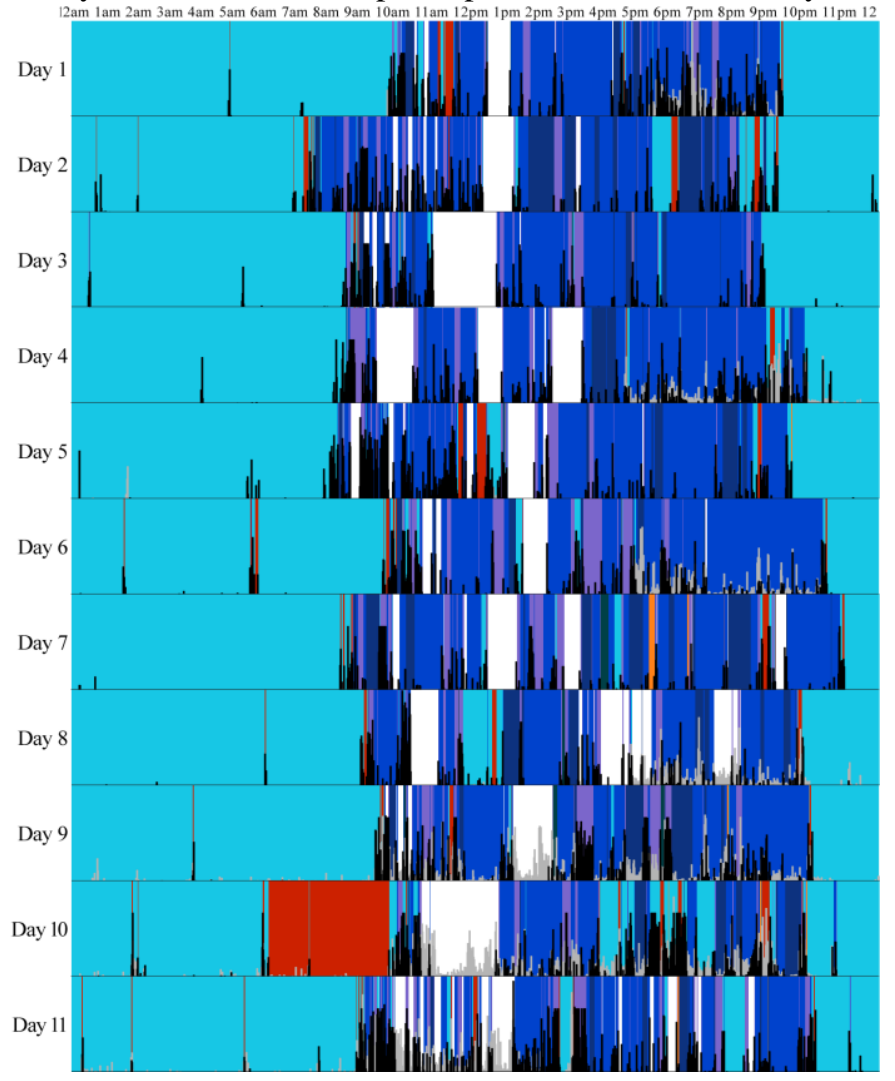

Figure 6. Location map for participant 3B, a good sleeper (PSQI=1) movement at night than participant $2 \mathrm{~B}$. This is reflected in their PSQI sleep quality scores, with participant 3B scoring 1 (good) in contrast to a score of 12 (poor) for participant 2B. Conversely the bed time of participant $1 \mathrm{~B}$ is variable, as late as between $3 \mathrm{am}$ and $4 \mathrm{am}$. Rise time also appears variable, with participant $2 \mathrm{~B}$ occasionally staying in the bedroom until $1 \mathrm{pm}-2 \mathrm{pm}$. Movement at night is also relatively high for participant IB. While a PSQI score of 6 is not as high as that of participant $2 \mathrm{~B}$, participant $1 \mathrm{~B}$ is still determined to be a poor sleeper. When considering time outside the home, participant $1 \mathrm{~B}$ spends elongated periods outside their home, almost every day, sometimes twice per day. Whilst participant 3B also spends a considerable amount of time outside the home, he tends to spend short durations outside regularly throughout the day, and frequently goes out around lunchtime. In contrast participant $2 \mathrm{~B}$ spends very little time outside the home, aside from on day 7 when he was away for over an hour at lunchtime and several hours that night, delaying his usually regular bed time.

\section{LONGITUdinal ANALYSIS OF THE DERIVED BeHAVIOUR METRICS}

This section compares the objective measures for time in bed (TIB), total movement (TM) and time outside the home (TOTH) of participants $1 \mathrm{~B}, 2 \mathrm{~B}$ and $3 \mathrm{~B}$, over an extended duration between April 2011 and January 2013 (totalling 22 months). These metrics have been derived using the validated location mapping model, as described in section III, and results are presented in fig. 7 and Table II. Variability is evident between each resident for the period.

In analysing weekly averages, illustrated in fig. 7, participant 1B experiences an overall decrease in TIB over the period of analysis, starting at an average of $10 \mathrm{hrs}$ and 6 mins (10.10 hrs) in April 2011 and lowering to $7 \mathrm{hrs}$ and $23 \mathrm{mins}$ (7.38 hrs) in January 2013. This participant spends a considerable amount of time outside the home, as much as 10 hours per day, peaking between December and February, as shown in table II. This gradual increase in TOTH coincides with a decrease in loneliness, with the participant scoring 4 (moderately lonely) on the De Jong scale of emotional and social loneliness in June 2012 in contrast to a score of 1 (not lonely) in November 2012. While TM is low for participant $1 \mathrm{~B}$ in comparison to participants $2 \mathrm{~B}$ and $3 \mathrm{~B}$ it has increased from 23 mins ( $0.38 \mathrm{hrs)}$ to 1 hour 24 mins (1.4 hrs) over the duration of analysis. Their relatively low TM may result from the comparably high TOTH.

Although participant 2B reports poor subjective sleep quality, objective results portray a strong routine, particularly from January 2012 onwards, with a monthly average of between 8.5 and 10 hours TIB. However in some cases high weekly averages of up to $11 \mathrm{hrs} 13$ mins were recorded by both the ambient sensors and the actigraph, this may relate to a difficultly differentiating periods of quiescent wake in bed and sleep [27]. In such circumstances, long periods of quiescent wake, resulting in a high TIB, would not be attributed to a high quality of sleep. In contrast to the other participants, TOTH is very low, aside from a peak in October 2012, which may be attributed to the participant returning late at night from a holiday. Despite the low TOTH, TM is not significantly 
(a)

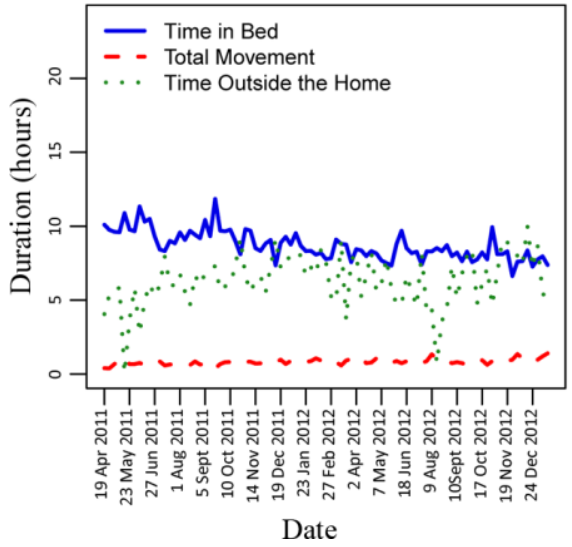

(b)

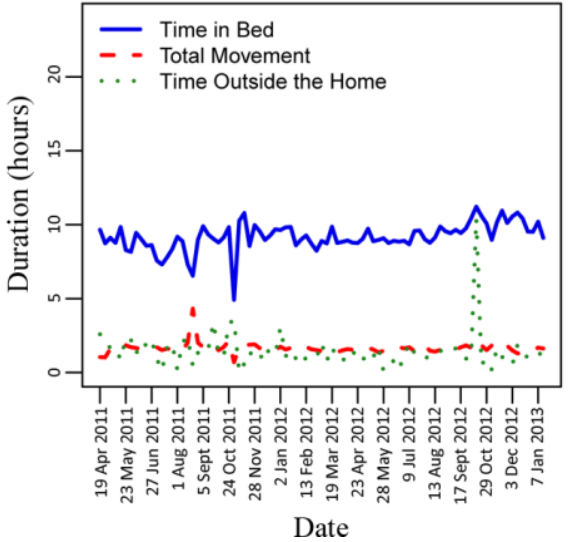

(c)

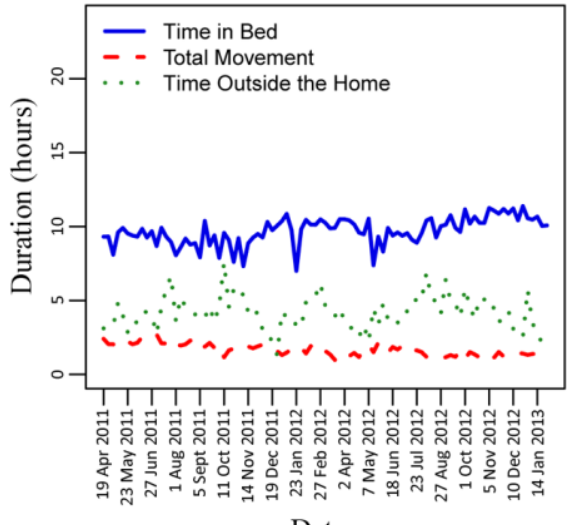

Figure 7. Weekly averages for participants 1B (a), 2B (b), and 3B (c), with PSQIs of 6, 12 and 1 respectively, between April 2011 and January 2013

more than the other residents. The low TOTH may be associated with an increase in loneliness from a score of 7 (moderately lonely) in July 2012 to a score of 9 (severely lonely) in November 2012.

Participant 3B appears to have a relatively varied TIB, with a general increase experienced during the winter months. A sharp decrease in TIB is apparent in late December 2011/early January 2012, dipping from a weekly average of 10 hours 32 mins ( $10.54 \mathrm{hrs})$ to 7 hours 19 mins $(7.38 \mathrm{hrs})$, coinciding with an increase in TOTH, increasing from a weekly average of 2 hours $14 \mathrm{mins}$ (2.24 hrs) to 4 hours $29 \mathrm{mins}$ (4.48), in comparison to the weeks previous. While there seems to be a gradual increase in TIB overall, rising from an average of 8-9 hours per night to 10-11 hours, a gradual decrease in TM captured by the PIRs is evident. This decrease in TM may result from an increase in TIB or TOTH. Their TIB tends to increase slightly during the winter months as illustrated in table II. This change is confirmed in their PSQI questionnaire, with sleep duration increasing from 8 hours in June 2012 to 11.5 hours in November 2012.

\section{DISCUSSION AND CONCLUSIONS}

Wrist actigraphy compliance was found to be a concern when carrying out the validation of group $B$, with participant $2 \mathrm{~B}$ removing the actigraph for one night and participant $3 \mathrm{~B}$ appearing to take the actigraph off for seven out of ten nights, leaving only three nights of valid data. This indicates that actigraphy may not be a viable solution to objectively monitor sleep measures longitudinally. Aside from compliance in wearing the actigraphs, not all participants used the marker button to indicate their bed and rise times. In these cases location maps proved useful in determining the window within which to check for sleep using the actigraphy software. The completion of sleep diaries was also inconsistent with one participant requiring assistance. Consequently, sleep diaries were filled in every $2 / 3$ days during their validation period.

The overshoot time of PIRs installed in GNH may result in mislabelling in the location mapping algorithm when sensors, used to detect movement to adjacent rooms, are located in direct view of a PIR. This is evident on day 10 for participant $3 \mathrm{~B}$, illustrated in fig. 6 , in which the resident woke up, visited

TABLE II. Seasonal Averages By Month For Participants 1B, 2B AND 3B BETwEen April 2011 AND January 2013

\begin{tabular}{|c|c|c|c|c|c|c|c|c|c|c|c|c|c|}
\hline \multirow[b]{2}{*}{$\vartheta$} & Sensor-Derived Time in Bed (TIB-S) & 8.62 & 8.23 & 8.39 & 8.76 & 8.65 & 9.85 & 8.35 & 8.92 & 8.94 & 8.88 & 8.42 & 8.19 \\
\hline & Time Outside the Home (TOTH) & 7.26 & 7.27 & 5.93 & 6.31 & 5.62 & 5.19 & 6.04 & 5.18 & 6.90 & 6.41 & 7.18 & 7.91 \\
\hline \multirow{3}{*}{ สิ } & Sensor-Derived Time in Bed (TIB-S) & 9.68 & 8.86 & 8.88 & 8.96 & 8.99 & 8.86 & 8.53 & 8.9 & 9.51 & 9.79 & 9.86 & 9.86 \\
\hline & Time Outside the Home (TOTH) & 1.36 & 0.90 & 1.43 & 1.45 & 1.40 & 1.35 & 1.30 & 1.34 & 1.59 & 1.61 & 0.94 & 1.21 \\
\hline & Total Movement (TM) & 1.64 & 1.60 & 1.48 & 1.40 & 1.67 & 1.62 & 1.59 & 1.76 & 1.75 & 1.72 & 1.79 & 1.54 \\
\hline लै & Sensor-Derived Time in Bed (TIB-S) & 10.02 & 10.17 & 10.15 & 9.91 & 9.34 & 9.44 & 9.23 & 9.29 & 9.66 & 9.54 & 9.81 & 10.4 \\
\hline
\end{tabular}


the en suite (red), closed the door and went back to bed (aqua). Location detection has been established to be most accurate when the placement of the bed is within the dark green areas portrayed in fig. 1. Such uncertainty is caused by not having full PIR visibility.

This paper presents a method of detecting measures of night time behaviour, including bed time, rise time, time in bed, total movement and time outside the home. A system of ambient sensors installed in apartments in Great Northern Haven was rigorously validated and compared on a nightly basis with comparable measures derived from actigraph data for five adults staying in the test/demonstration apartment for between 2 and 4 nights as well as three individuals living in their own homes. Sensor-derived metrics have been validated for a number of individuals, with differing sleep problems and health status, residing in GNH. A longitudinal analysis was undertaken, relating time in bed to time outside the home and movement levels detected by the three PIRs installed in each home. Longitudinal, as well as seasonal, variability was evident for two individuals in particular. The third individual analysed has very little variability in time spent outside the home, as well as low levels of movement detected by the PIRs. This indicates the importance of monitoring individuals, based on their own normal behaviour. While the layout of GNH apartments is identical throughout the complex, the technique presented may be extended to any singly occupied home, providing PIRs installed detect room transitions. Future directions for research will include the comparison of clinical health assessments with automated activity recognition algorithms.

\section{REFERENCES}

[1] P. Emery, "Evidence supporting the benefit of early intervention in rheumatoid arthritis," The Journal of Rheumatology, vol. 66, pp. 3-8, 2002.

[2] V. O. C. Rialle, C. Guigui and C. \& Hervé, "What do family caregivers of Alzheimer's disease patients desire in smart home technologies? Contrasted results of a wide survey," Methods Information in Medicine, vol. 47, pp. 63-69, 2008.

[3] W. R. Swindell, et al., "Indicators of "healthy aging" in older women (65-69 years of age). A data-mining approach based on prediction of long-term survival," BMC Geriatrics, vol. 10, p. 55, 2010.

[4] S. Happe, "Excessive daytime sleepiness and sleep disturbances in patients with neurological diseases," Drugs vol. 63, pp. 2725-2737, 2003.

[5] N. Stanley, "The physiology of sleep and the impact of ageing," European Urology Supplements, vol. 3, pp. 17-23, 2005.

[6] D. J. Buysse, C. F. Reynolds, T. H. Monk, S. R. Berman and D. J. Kupfer, "The Pittsburgh sleep quality index: a new instrument for psychiatric practice and research," Psychiatry Research, vol. 28, pp. 193213, 1989.

[7] J. Choi, B. Kim, B. Hwang, R. Sohn and K. Park, "Unobtrusive body movement monitoring during sleep using infrared motion detector and ZigBee protocol," in IEEE EMBS, 2006, pp. 32-33.

[8] J. Shin, Y. Yoon and J. C. Principe, "Evaluation of body movement during sleep by thermopile using wavelet and neuro-fuzzy," in IEEE EMBC, 2003, pp. 2406-2407.
[9] P. De Chazal, et al., "Sleep/wake measurement using a non - contact biomotion sensor," Journal of sleep research, vol. 20, pp. 356-366, 2011.

[10] L. Walsh, E. Moloney and S. McLoone, "Identification of nocturnal movements during sleep using the non-contact under mattress bed sensor," in IEEE EMBC, 2011, pp. 1660-1663.

[11] Z. T. Beattie, C. C. Hagen and T. L. Hayes, "Classification of lying position using load cells under the bed," in IEEE EMBC, 2011, pp. 474477.

[12] Z. T. Beattie, C. C. Hagen, M. Pavel and T. L. Hayes, "Classification of breathing events using load cells under the bed," in IEEE EMBC, 2009, pp. 3921-3924.

[13] W. Chen, et al., "Unconstrained monitoring of long-term heart and breath rates during sleep," Physiological Measurement, vol. 29, p. N1, 2008.

[14] M. J. Rantz, M. Skubic and S. J. Miller, "Using sensor technology to augment traditional healthcare," in IEEE EMBC, 2009, pp. 6159-6162.

[15] X. Zhu, W. Chen, Z. Tang, T. Nemoto and D. Wei, "Automatic home care system for monitoring HR/RR during sleep," in IEEE EMBS, 2008, pp. 522-525.

[16] T. Hayes, M. Pavel and J. Kaye, "An unobtrusive in-home monitoring system for detection of key motor changes preceding cognitive decline," in IEEE EMBS, 2004, pp. 2480-2483.

[17] M. Pavel, T. Hayes, A. Adami, H. Jimison and J. Kaye, "Unobtrusive assessment of mobility," in IEEE EMBS, 2006, pp. 6277-6280.

[18] A. Adami, T. Hayes and M. Pavel, "Unobtrusive monitoring of sleep patterns," in IEEE EMBS, 2003, pp. 1360-1363.

[19] P. Rashidi, D. J. Cook, L. B. Holder and M. Schmitter-Edgecombe, "Discovering activities to recognize and track in a smart environment," IEEE Transactions on Knowledge and Data Engineering, vol. 23, pp. 527-539, 2011

[20] D. J. Cook, N. C. Krishnan and P. Rashidi, "Activity Discovery and Activity Recognition: A New Partnership," IEEE Transactions on Cybernetics, vol. PP, pp. 1-9, 2013.

[21] M. J. Rantz, et al., "TigerPlace: A new future for older adults," Journal of Nursing Care Quality, vol. 20, pp. 1-4, 2005.

[22] S. Wang and M. Skubic, "Density map visualization from motion sensors for monitoring activity level," in IET, 2008, pp. 1-8.

[23] S. Wang, M. Skubic, Y. Zhu and C. Galambos, "Using passive sensing to estimate relative energy expenditure for eldercare monitoring," in PERCOM, Seattle, WA, 2011, pp. 598-604.

[24] M. J. Rantz, et al., "TigerPlace, a State-Academic-Private project to revolutionize traditional Long-Term care," Journal of Housing for the Elderly, vol. 22, pp. 66-85, 2008.

[25] J. de Jong-Gierveld and F. Kamphuls, "The development of a Raschtype loneliness scale," Applied Psychological Measurement, vol. 9, pp. 289-299, 1985.

[26] A. O' Brien, K. McDaid, J. Loane, J. Doyle and B. O'Mullane, "Visualisation of Movement of Older Adults within their Homes based on PIR Sensor Data," in PERVASENSE, San Diego, CA, 2012.

[27] J. Paquet, A. Kawinska and J. Carrier, "Wake detection capacity of actigraphy during sleep," Sleep, vol. 30, pp. 1362-1369, 2007. 